\title{
AN ABELIAN $p$-GROUP WITHOUT THE ISOMORPHIC REFINEMENT PROPERTY ${ }^{1}$
}

\author{
BY A. L. S. CORNER AND PETER CRAWLEY
}

Communicated by R. S. Pierce, March 1, 1968

It is well known that a countable reduced abelian $p$-group $G$ has the isomorphic refinement property, i.e. any two direct decompositions of $G$ have isomorphic refinements, if and only if $G$ has no elements of infinite height. In the uncountable case the situation is characteristically unclear. There do exist uncountable reduced $p$ groups with nonzero elements of infinite height having the isomorphic refinement property; any $p$-group whose first Ulm factor is torsioncomplete and second Ulm factor is cyclic is such a group. And for uncountable $p$-groups with no elements of infinite height there exist sufficient conditions for the isomorphic refinement property, e.g., those of Crawley [3] and Warfield [4]. Yet the question has remained whether the isomorphic refinement property is possessed by all such groups. Here we answer this question in the negative by showing that there exists an abelian p-group with no elements of infinite height having two direct decompositions that do not admit isomorphic refinements.

The foregoing result is actually obtained as a corollary to the following theorem: there exist three abelian p-groups $K, L$ and $M$, each with no elements of infinite height, such that no Ulm invariant of $K$ exceeds $1, K \oplus L \cong K \oplus M$, yet $L \not M$. In particular, this shows that the cancellation theorem of Crawley [2] does not extend to the uncountable case.

To see how our first result follows from the second, let $K, L$ and $M$ be as above, and assume further that $K$ has the isomorphic refinement property. We will show that the direct decompositions $K \oplus L \cong K \oplus M$ do not have isomorphic refinements. If they do, there exist groups $K_{i}, L_{i}, K_{i}^{\prime}, M_{i}(i=1,2)$ such that

$$
K=K_{1} \oplus K_{2}=K_{1}^{\prime} \oplus K_{2}^{\prime}, \quad L=L_{1} \oplus L_{2}, \quad M=M_{1} \oplus M_{2},
$$

and

$$
K_{1} \cong K_{1}^{\prime}, \quad K_{2} \cong M_{1}, \quad L_{1} \cong K_{2}^{\prime}, \quad L_{2} \cong M_{2} .
$$

Now by assumption, $K$ has the isomorphic refinement property, and therefore there exist groups $K_{i j}, K_{v j}^{\prime}(i, j=1,2)$ such that

1 This work was supported in part by NSF Grant GP 7252. The authors are currently at Vanderbilt University. 


$$
K_{i}=K_{i 1} \oplus K_{i 2}, \quad K_{i}^{\prime}=K_{i 1}^{\prime} \oplus K_{i 2}^{\prime} \quad(i=1,2),
$$

and

$$
K_{i j} \cong K_{j i}^{\prime} \quad(i, j=1,2) .
$$

But all the Ulm invariants of $K$ are at most 1 , and $K_{1} \cong K_{1}^{\prime}$, so $K_{1}$ can have no nonzero Ulm invariant in common with either $K_{2}$ or $K_{2}^{\prime}$. Therefore we must have

$$
K_{12}=K_{21}^{\prime}=K_{21}=K_{12}^{\prime}=0,
$$

and consequently $K_{2}=K_{22} \cong K_{22}^{\prime}=K_{2}^{\prime}$. This implies that $L_{1} \cong M_{1}$, and we infer that $L=L_{1} \oplus L_{2} \cong M_{1} \oplus M_{2}=M$, a contradiction. Thus if $K$ has the isomorphic refinement property, then $K \oplus L$ does not.

Throughout the proof of the second result the following notation is used. The cyclic subgroup generated by an element $x$ in an abelian $p$-group $G$ is denoted by $[x]$, and $G\left[p^{n}\right]$ and $p^{n} G$ denote, respectively, the subgroups of those element of order at most $p^{n}$ and those elements of height at least $n . E(G)$ denotes the ring of endomorphisms of the group $G$, and endomorphisms are written on the right. An endomorphism $\phi \in E(G)$ is called small if for each integer $e>0$ there exists an integer $n>0$ such that $\left(p^{n} G\right)\left[p^{e}\right] \phi=0$. The small endomorphisms form an ideal of $E(G)$ which we denote by $E_{s}(G)$. Finally $Z$ denotes the ring of integers.

Our proof requires the following theorem of Corner [1]. Let $\bar{B}$ be a torsion-complete abelian p-group with an unbounded countable basic subgroup $B$, and let $\Phi$ be a separable closed subring of $E(\bar{B})$ in the $p$-adic topology. Suppose further that $\Phi$ satisfies the condition

(C) if $\phi \in \Phi$ and $\left(p^{n} \bar{B}\right)[p] \phi=0$ for some integer $n>0$, then $\phi \in p \Phi$. Then there exists a pure subgroup $G$ of $\bar{B}$ which contains $B$, such that $E(G)=\Phi \oplus E_{0}(G)$.

For our purposes here, let $A$ be a direct sum of cyclic groups, $A=\sum_{n<\infty}\left[a_{n}\right]$, where each $a_{n}$ has order $p^{n}$. Set $B=A \oplus A$, and let $\bar{A}$ and $\bar{B}$ be, respectively, the torsion-completions of $A$ and $B$. Let $\tau$ be that endomorphism of $\bar{A}$ defined by the rule

$$
a_{n} \tau=p a_{n+1} \quad(n=1,2, \cdots),
$$

and let $R$ be the subring of $E(\bar{A})$ generated by $\tau$. Trivially $R \cong Z[\tau], \tau$ transcendental. Identify the ring of all $2 \times 2$ matrices over $R$ as a subring of $E(\bar{A} \oplus \bar{A})=E(\bar{B})$, and let $S$ be the subring of all matrices of the form

$$
\left(\begin{array}{cc}
f_{11}(\tau) & \left(1-\tau^{2}\right) f_{12}(\tau) \\
f_{21}(\tau) & a+\left(1-\tau^{2}\right) f_{22}(\tau)
\end{array}\right) \quad a \in Z, f_{i j}(\tau) \in R
$$


The ring $S$ contains the matrices

$$
\begin{array}{ll}
\theta=\left(\begin{array}{cc}
\tau & 1-\tau^{2} \\
0 & 0
\end{array}\right), & \phi=\left(\begin{array}{ll}
\tau & 0 \\
1 & 0
\end{array}\right), \\
\alpha=\theta \phi=\left(\begin{array}{ll}
1 & 0 \\
0 & 0
\end{array}\right), & \beta=\phi \theta=\left(\begin{array}{cc}
\tau^{2} & \tau\left(1-\tau^{2}\right) \\
\tau & 1-\tau^{2}
\end{array}\right) ;
\end{array}
$$

and $\theta \phi \theta=\theta, \phi \theta \phi=\phi$. Consequently $\alpha$ and $\beta$ are equivalent idempotents in $S$. Let $\bar{R}$ and $\bar{S}$ be, respectively, the $p$-adic closures of $R$ in $E(\bar{A})$ and $S$ in $E(\bar{B})$. It is easy to check that the ring $\bar{R}$ satisfies condition $(C)$ with $\bar{B}$ replaced by $\bar{A}$, and from this and the purity of $\bar{S}$ in $E(\bar{B})$ it follows that $\bar{S}$ satisfies $(C)$. Therefore by the theorem above there exists a pure subgroup $G$ of $\bar{B}$ which contains $B$ and such that $E(G)=\bar{S} \oplus E_{s}(G)$.

Since $\alpha$ and $\beta$ are equivalent idempotents in $E(G)$,

$$
G=G \alpha \oplus G(1-\alpha)=G \beta \oplus G(1-\beta)
$$

and $G \alpha \cong G \beta$. Moreover, $B \alpha$ is a basic subgroup of $G \alpha$, and $A \cong B \alpha$, so that the $n$th Ulm invariant of $G \alpha$ is 1 for each $n=0,1,2, \cdots$. Suppose that $G(1-\alpha) \cong G(1-\beta)$. Then $1-\alpha$ and $1-\beta$ are equivalent idempotents in $E(G)$ and hence in $\bar{S}$, i.e. there exist endomorphisms $\mu, \nu \in \bar{S}$ with $\mu \nu=1-\alpha, \nu \mu=1-\beta, \mu \nu \mu=\mu, \nu \mu \nu=\nu$. Reduce all matrices in sight modulo $p$. Since $\alpha \mu=\nu \alpha=0$, we may set

$$
\mu=\left(\begin{array}{cc}
0 & 0 \\
f_{1}(\tau) & a+\left(1-\tau^{2}\right) f_{2}(\tau)
\end{array}\right), \quad \nu=\left(\begin{array}{cc}
0 & g_{1}(\tau) \\
0 & b+\left(1-\tau^{2}\right) g_{2}(\tau)
\end{array}\right)
$$

where $a, b \in Z / p Z$ and $f_{i}(\tau), g_{i}(\tau)$ are polynomials in $\tau$ over $Z / p Z$. Equate respectively the $(1,1)$ and $(2,1)$ entries in $\nu \mu=1-\beta$ to obtain

$$
g_{1}(\tau) f_{1}(\tau)=1-\tau^{2}, \quad\left(b+\left(1-\tau^{2}\right) g_{2}(\tau)\right) f_{1}(\tau)=-\tau .
$$

The second equation requires that $g_{2}(\tau)=0$, so that $\tau \mid f_{1}(\tau)$, and this formula combined with the first equation gives $\tau \mid 1-\tau^{2}$, a contradiction. Therefore $G(1-\alpha) \nsucceq G(1-\beta)$, and the proof is complete.

\section{REFERENCES}

1. A. L. S. Corner, On endomorphism rings of primary abelian groups, (to appear).

2. P. Crawley, The cancellation of torsion abelian groups in direct sums, J. of Algebra 2 (1965), 432-442.

3. - An isomorphic refinement theorem for certain abelian p-groups, J. of Algebra 6 (1967), 376-387.

4. R. B. Warfield, Jr., Complete abelian groups and direct sum decompositions, Thesis, Harvard University, Cambridge, Mass., 1967.

Worcester COLlege, OXFord, AND

California Institute of Technology 